\title{
Contamination Analysis and Monitoring Methods of Hydraulic Fluid Rui ZENG ${ }^{1,2}$, Yong ZHANG ${ }^{2}$, Zhen-Rong LIN ${ }^{2}$ and Jin-Kun SUN ${ }^{2}$ \\ ${ }^{1}$ College of Field Engineering, PLA Univ. of Sci. \& Tech., Nanjing 210007, China \\ 2 Department of Airfield Engineering, Air Force Logistics College, Xuzhou 221000, China \\ Corresponding author: zri1980@126.com
}

Keywords: Contamination, Hydraulic system, Monitoring method, Hydraulic fluid, Pollution control standard.

\begin{abstract}
In recent years, with the development of construction machinery technology, the controlling requirement for its hydraulic system has become more and more strict. The bad working environments did harm for the hydraulic system, so people payed more attention on preventing hydraulic system from invalid and fault. The experience and research showed that hydraulic fluid contamination was the main reason for the invalid and fault of construction machinery. This paper made detailed analysis for the contamination problem, concluded the monitoring methods for the contamination of hydraulic system, and discussed its research emphases and developments. With the development of the research work and the continuous improvement of the monitoring method, the pollution of the hydraulic system will be effectively controlled, so that the pollution degree of the hydraulic oil pollution can be kept in the range of pollution and tolerance.
\end{abstract}

\section{Introduction}

According to its physical state, contamination of engineering machinery hydraulic system in the oil can be divided into solid state, liquid and gas[1] Three types of solid pollutants usually exist in the system with granular oil liquid. Liquid pollutants are mainly outside intrusion system water and gaseous pollutants are mainly air. The source of contamination in the system oil is generally in the following aspects:

(1) Sand, chips, abrasive, rust and dust have been brought into before the hydraulic system worked.

(2) During the work of the hydraulic system, through the reciprocating movement of the piston rod, oil system, air tank, splash droplets of oil in the tank, etc. the dust intrusion system.

(3) In the process of the work, the new metal, airtight material wear particles, the filter material falling off of the particle fiber, peeling of the paint slag and so on.

(4) In the process of maintenance, the dust and lint are carried into.

(5) Oil metamorphism produces chemical reaction, and makes the metal corrosion, rust particles, slice.

\section{Oil Pollution Testing Standards}

International generally used the NAS as a pollution control standards conference. Comparing economic is using blood count method, which is generally only four times, and accuracy can reach above 95\%[2]. Also can use weight calibration, which allow particles in $100 \mathrm{ml}$ of oil weight said. Measurement method is to $100 \mathrm{ml}$ of oil after 0.45 microns diameter of membrane filtration, drying, in the film balance weight. Construction machinery oil controls the general level of 101-102.

In fact, different hydraulic systems of different sizes within the scope of the tolerance is not the same, and the pollution of a certain particle size within the scope of the pollution is the main cause of failure. The size is called sensitive size[3]:

For a practical hydraulic system, the biggest harm is the pollution particles distributed in the nearby sensitive size range. These particles should be the main control object of oil pollution 
control, pollution grade code only maximum as the degree of contamination of the final results of the evaluation is the degree of harm of oil pollution the solution cannot be objectively described.

The effect of various factors on the oil pollution in a specific environment is not the same, only with a single factor to determine the degree of pollution can not accurately determine the extent of the damage. In order to solve this problem, the two methods are commonly used in hierarchical fuzzy comprehensive evaluation and weighted correlation analysis.

\section{Hierarchical Fuzzy Comprehensive Evaluation Method}

Given two finite fields as follows :

$$
U=\left\{u_{1}, u_{2}, u_{3}, \cdots \cdots, u_{n}\right\} \quad V=\left\{v_{1}, v_{2}, v_{3}, \cdots \cdots, v_{m}\right\}
$$

The collection of $U$ representative factors comprehensive evaluation form for hydraulic oil, physical and chemical properties, its elements: viscosity, flash point, flash point, density, degree of pollution, should depend on the specific requirements of the system and use $V$; set represents the evaluation objects consisting of several oil elements for the poor the degree of different. The single factor fuzzy evaluation vector of the set of $M$ objects, which is set up as the first factor, can be regarded as a fuzzy subset on the object set $V$, which indicates the membership degree of the first $\mathrm{j}$ evaluation object to the I factor, and the fuzzy relation matrix is obtained:

$$
R=\left[r_{i j}\right] \quad i=1,2 \cdots n ; j=1,2 \cdots m
$$

According to the importance of the evaluation factors of oil performance, establish the evaluation factor weight vector $A$ :

$$
A=\left\{a_{1}, a_{2}, a_{3}, \cdots \cdots, a_{n}\right\}
$$

Obviously, A is a fuzzy subset of the $\mathrm{U}$, which is the evaluation of the factors weights. Usually, the weights satisfy the normalization condition:

$$
\sum_{i=1}^{n} a_{i}=1 \quad\left(a_{i}>0\right)
$$

Let $S$ to judge the result vector, and introduce the fuzzy transformation:S=AR.

$\mathrm{S}$ is a fuzzy subset of $V$, when the fuzzy relation matrix $R$ as fuzzy evaluation, fuzzy input weight vector $A$, the formula for the evaluation results of vector $\mathrm{S}$ (output vector), it is a kind of fuzzy transform fuzzy comprehensive evaluation problem.

The fuzzy transformation operator according to the maximum, minimum algorithm, but the maximum and minimum operation only highlights the minimum membership degree, in the actual problem is not reasonable. Therefore, fuzzy comprehensive evaluation method not using linear transformation method by ordinary matrix addition, multiplication. That is:

$$
\begin{gathered}
S=A R=A\left[r_{i j}\right]=\left(s_{1}, s_{2}, s_{3}, \cdots \cdots, s_{m}\right) \\
s_{j}=\sum_{k=1}^{n} a_{k} r_{k j} \quad(j=1,2, \cdots m)
\end{gathered}
$$

Let $V t$ be a new oil (standard) oil, $V i$ oil for different degree of degradation.

The relative deterioration index vector of the oil properties can be defined as $X$ :

$$
X_{i}=\left|S_{i}-S_{t}\right| / S_{t} \quad(i=2,3, \cdots m)
$$

The smaller the $X$, the smaller the degree of deterioration of the oil performance.

If you set the $\varepsilon$ for the comprehensive performance of the oil change is the allowable limit, the oil condition of hydraulic system for $X>\varepsilon$.

$$
V_{\varepsilon}=\left\{V_{i} \mid X_{i}>\varepsilon, i=1,2, \cdots, m\right\}
$$


The above is the mathematical model of fuzzy comprehensive evaluation in practice according to the evaluation results of vector $S$ each element size, can determine the pros and cons of each evaluation object, such as the maximum, the K oil samples. The best overall performance and so on, can be for the comprehensive performance of the pros and cons of each evaluation object queue according to the comprehensive evaluation of the results obtained. The failure of oil sample set can be set to provide guidance for the hydraulic system of the oil.

\section{Weighted Correlation Analysis Method}

With two series, $x_{i}(k)$ and $x_{j}(k)$ are two series in the $K$ point of the numerical value, then:

$$
\varepsilon_{i j}(k)=\frac{\Delta_{\min }+\rho \Delta_{\max }}{\Delta_{i j}(k)+\rho \Delta_{\max }}
$$

$\varepsilon_{i j}(k)$ for the two series in the $K$ point of the gray correlation coefficient; $\Delta_{\max }, \Delta_{\min }$ respectively, the absolute difference between the point of the maximum and the minimum value; $\rho$ for the resolution factor. $\Delta_{i j}(k)$ represents the absolute difference between the two values at the $K$ point.

$$
\Delta_{i j}(k)=\left|x_{i}(k)-x_{j}(k)\right|
$$

The grey relation degree is a kind of index to characterize the similarity between the two grey systems:

$$
\gamma_{i j}=\frac{1}{n} \sum_{k=1}^{n} \varepsilon_{i j}(k)
$$

In formula, $n$ is the length of the two series; $r$ is the grey relational degree.

In the engineering system, and the related factors in the system's position is different, so the weight of each factor is different. Therefore, for grey relational analysis, can take different weights among various factors, to take a weighted coefficient.

$$
\gamma_{i j} \cdot \sum_{k=1}^{n} \beta_{k} \cdot \varepsilon_{i j}(k)
$$

Assume the use of the 5 dimensional range of the test results as a reference sequence:

$$
X_{0}=\left\{x_{0}(1), x_{0}(2), \cdots \cdots x_{0}(5)\right\}
$$

The number of particles in the range of particle size of the 5 size range specified by the NAS1638 grade standard is compared with the number of sequences.

$$
X_{j}=\left\{x_{j}(1), x_{j}(2), \cdots \cdots x_{j}(5)\right\}
$$

So we can get the factor grey correlation coefficient matrix between the comparison sequence and the reference sequence.

$$
B_{\varepsilon}=\left[\begin{array}{cccc}
\varepsilon_{01}(1) & \varepsilon_{01}(2) & \cdots \cdots & \varepsilon_{01}(1) \\
\varepsilon_{02}(1) & \varepsilon_{02}(2) & \cdots \cdots & \varepsilon_{02}(5) \\
\cdots \cdots & \cdots \cdots & \cdots \cdots & \cdots \cdots \\
\varepsilon_{014}(1) & \varepsilon_{014}(2) & \cdots \cdots & \varepsilon_{014}(5)
\end{array}\right]
$$

The weight distribution should be near a particle size range of pollution sensitive. Because the hydraulic system is composed of various hydraulic elements, sensitive size may be in different size ranges, then according to the degree of importance of components by weight allocation into consideration. In practical application, the choice of the weights should be studied according to the actual situation. Considering various factors, selecting a group of appropriate weight, weight matrix:

$$
A_{\beta}=\left(\beta_{1}, \beta_{2}, \beta_{3}, \beta_{4}, \beta_{5}\right),\left(\beta_{k}>0, k=1,2,3,4,5\right)
$$




$$
\sum_{k=1}^{5} \beta_{k}=1
$$

The weight matrix and gray correlation coefficient matrix of factor substitution, weighted grey correlation matrix:

$$
P_{\varepsilon}=A_{\beta} \cdot B_{\varepsilon}=\left[\gamma_{01} \cdot \gamma_{02} \cdot \cdots, \gamma_{014} \cdot\right]
$$

\section{Detection Method of Oil Pollution}

All kinds of particulate pollutants generated inside hydraulic and machine lubrication system, with a large number of reflecting the state of the system of internal information, through the identification of oil particle contamination, can detect the contamination wear of hydraulic components or machine parts, and provide important clues and basis for fault diagnosis of hydraulic system and mechanical equipment. At present, the main methods of identifying the components of particulate pollutants are: spectral analysis, $X$ ray analysis and iron spectrum analysis [4].

The solid particles are the most important and harmful pollutants in oil, and the oil pollution is mainly aimed at the solid particles. Oil contamination monitoring methods are: visual colorimetric monitoring, congestion index check weighing method monitoring, electronic counter and particle optical particle counting method.

Detection of water content in mineral oil is also an important aspect of pollution control of hydraulic and lubrication systems. The percentage of water content in oil is generally expressed as a percentage of trace water.

According to the water content in the oil and the accuracy of the determination results, the determination method of water content in oil is: distillation method, Carle's method and semi quantitative method.

Hydraulic and lubricating oil systems are more or less air containing. The oil in the air has three states: free air bag or large bubbles, small bubbles of uniform suspension, and the air dissolved in the oil. There is a close relationship between the state and the characteristics of different liquid and system, in need of determination of air content in the oil system can adopt the following methods: oil appearance detection method, turbidimetric method, ultrasonic method, vacuum degassing method, volume compression method

\section{Basic Control Method of Oil Pollution}

Hydraulic and lubrication system in the work, the external contaminants continue to invade the system, while the internal system and continuously produce pollutants, these pollutants on the system's reliability and the life of components by a direct impact.

In order to ensure the normal operation of the system, it is necessary to take effective measures to clean up all kinds of pollutants in the oil to keep the cleanliness of the oil.

According to the different pollutants and purification of oil, can adopt different purification methods, these methods include filtration, centrifugation, coalescence, electrostatic, magnetic, vacuum and adsorption.

\section{Reduce Latent Dirt}

(1) The degree of pollution components check strictly. Pump, valve, high pressure hose, cylinder and other main components for purchased parts, must request to complete the factory. Transportation and storage process, all the oil port are sealed, prevent dirt into.

(2) Before the assembly, all components and accessories must be carefully cleaned before assembly of high temperature and high pressure, can clean the cavity. At a corner of the iron can use magnets to pull out. After cleaning, closed all the oil with plastic plugs.

(3) Strengthen the management of oil, oil into the plant must be sampled and tested, the test has to be filtered before the filter can be injected into the storage tank. 


\section{Prevent Dirt from Invading}

(1) To prevent environmental pollution, conditional assembly shop is best to be able to fill pressure, so that the indoor pressure is higher than the outdoor pressure, to prevent dust pollution in the atmosphere.

(2) All operations are using lubricants or cleaning fluid; the assembly in parts of the surface cleaning liquid from time to time in order to affect the quality of assembly, application of dry compressed air dry and then assembled.

(3) Element shall be presence test, loading, running and cleaning. High pressure hydraulic test bench oil than the oil pollution of the host system, oil contaminated and intrusive pollution on components. To set up multi-stage filtration hydraulic test system, the amount of pollution to the filter, the filter core is easy to disassemble and convenient regular cleaning. When using more than limit oil, oil should be timely.

\section{Prevent New Dirt}

The new hydraulic system in the dirt are mainly metal particles wear, system corrosion, paint peeling and high temperature oil deterioration. In order to prevent oil pollution, oil filter should be chosen properly. The general filtering precision and system components of precision should be adapted.

\section{Conclusion and Prospect}

(1) Direct access to images of particles in the oil, and the particle image processing, so as to get the size and distribution of particles, is the most effective and reliable method for obtaining oil contamination. Therefore, computer vision technology, analysis of microscopic imaging technology and particulate contamination of the fluid, is the focus of pollution monitoring of hydraulic machinery system engineering.

(2) The research and development of remote oil contamination on-line detecting system based on the principle of computer vision, is currently the subject of research focus and front. The system is composed of fluid sampling channel and computer image acquisition and processing of two parts. The fluid sampling channel consists of sampling pool, micro sampling pump and other components, can be oil on the tank or pipeline on-line sampling or off-line sampling of oil samples, driven by micro sampling pump, the oil slowly through the sample pool. The computer micro image acquisition and processing part includes the microscopic imaging system, CCD, image acquisition card and computer, display and so on. Oil pollution by sampling pool liquid microscopic imaging by microscopic imaging system convection, CCD image sensor, image signal detection, high-speed image acquisition card is converted to a digital signal, the digital image signal into the computer image processing and display. Finally using the wireless local area network technology, which has the function of remote monitoring.

(3) With the development of the research work and the continuous improvement of the monitoring method, the pollution of the hydraulic system will be effectively controlled, so that the pollution degree of the hydraulic oil pollution can be kept in the range of pollution and tolerance.

\section{References}

1. L H Gong, PLAS\&T, 11, 21(2002).

2. Q Z Tu, H F Zuo, Mst, 5,564(2004).

3. Q Z Tu, L H Gong, Q Wang, Mec,2,15(2002).

4. B F Gu, L H Gong, J H Gao. L\&S, 2,68(2004). 